\title{
Pedagogía no lineal en balonmano. Defensa zonal de una y dos líneas en partidos reducidos Non-linear pedagogy in handball. One and two lines zone defence in small-sided games
}

\author{
José Flores-Rodríguez, Gonzalo Ramírez-Macías
}

Universidad de Sevilla (España)

\begin{abstract}
Resumen. La pedagogía no lineal presenta diferencias importantes respecto a los modelos de enseñanza tradicionalmente empleados en balonmano. La ausencia de investigaciones sobre el proceso de enseñanza-aprendizaje en balonmano desde la pedagogía no lineal motivó el presente trabajo. El objetivo fue conocer la influencia ejercida por dos constreñimientos introducidos en una tarea: defensa zonal de una línea (LI1) y defensa zonal de dos líneas con defensor avanzado (LI2), en los comportamientos, ofensivos y defensivos, realizados por jugadores de balonmano. Estos constreñimientos, representativos de los sistemas defensivos más utilizados en balonmano, se aplicaron en la disputa de 16 partidos reducidos, durante las ocho sesiones en las que se desarrolló el estudio. Se utilizó un diseño observacional puntual/nomomético/multidimendional para registrar los comportamientos de 14 jugadores $(\mathrm{M}=14.6$ años y $\mathrm{SD}=0.4)$ de categoría cadete masculino, que se dividieron en dos grupos para la disputa de los partidos. El análisis de coordenadas polares permitió conocer la influencia ejercida por ambos constreñimientos a nivel general, analizando los comportamientos de ambos equipos conjuntamente y, también, de manera específica para cada equipo. Así, se encontraron relaciones significativas de activación mutua entre: (1) LI1 y fijación impar, lanzamiento en penetración, lanzamiento a distancia, bloqueo, cambio de oponente, ayudas y contrabloqueo; y (2) entre LI2 y desmarques, deslizamiento e interceptación. Conocer la influencia ejercida por los constreñimientos aplicados en las tareas (comportamientos que facilitan e inhiben) es clave en la aplicación de la pedagogía no lineal, de esta manera se podrán seleccionar aquellos que ayuden a conseguir los objetivos propuestos.
\end{abstract}

Palabras clave: Pedagogía deportiva, Juegos reducidos, Constreñimientos, Análisis de coordenadas polares, Iniciación deportiva.

\begin{abstract}
Non-linear pedagogy presents important differences regarding the teaching approaches traditionally used in handball. The absence of research that addresses the teaching-learning process in handball from nonlinear pedagogy motivated the present work. The objective was to analyse the influence of certain task constraints: zonal defense of one line (LI1) and zonal defense of two lines with advanced defender (LI2), in the behaviour patterns, offensive and defensive ones, performed by handball players. These constraints, representative of the most used defensive handball systems, were applied in 16 small-sided games, during the eight sessions in which the study was developed. A nomothetic/punctual/monitoring observational design was used to register the behaviours performed by 14 players $(\mathrm{M}=14.6$ years and $\mathrm{SD}=0.4)$, members of a team that competes in the male cadet category, who were divided into two groups for the matches. The analysis of polar coordinates allowed to know the influence exerted by both constraints at a general level, analyzing jointly the behaviors of both teams and, also, specifically for each team. Thus, significant relationships of mutual activation were found between: (1) LI1 and odd fixation, penetration throw, distance throw, block, opponent change, assist and counter block; and (2) between LI2 and uncheck, slip and intercept. Knowing the influence exerted by the task constraints (behaviors that facilitate and inhibit) is key in the application of non-linear pedagogy, in this way those that help to achieve the proposed objectives can be selected.
\end{abstract}

Keywords: Sport pedagogy, Small-sided games, Constraints, Polar coordinate analysis, Youth handball.

\section{Introducción}

La pedagogía no lineal presenta diferencias sustanciales respecto a los modelos de enseñanza tradicionalmente empleados en la enseñanza de los deportes colectivos, como el balonmano (Renshaw \& Chow, 2018). Estudios recientes indican que su utilización puede ofrecer mejores resultados que los obtenidos mediante la aplicación de metodologías conductistas del aprendizaje (Roberts, Rudd, Matthew \& Reeves, 2019). Basada en los postulados de la Psicología Ecológica y en la Teoría de los Sistemas Dinámicos (Chow, Davids, Button, Shuttleworth, Renshaw \& Araújo, 2007), presenta puntos en común con las teorías cognitivas del aprendizaje: el jugador asume un papel activo en el proceso de enseñanza-aprendizaje y utilizan juegos representativos del contexto real de juego que permiten al jugador encontrar la respuesta más adecuada (Práxedes, 2018). Aunque también presentan diferencias significativas. Las teorías cognitivas explican la acción deportiva según el modelo percepción-toma de decisión-ejecución, otorgando mucha importancia a los procesos conscientes y al conocimiento ex-

Fecha recepción: 01-05-20. Fecha de aceptación: 02-09-20 José Flores-Rodríguez

josefloresrodriguez@live.com plícito del jugador. Por su parte, la pedagogía no lineal asume una explicación de la acción deportiva basada en el ciclo percepción-acción (Chow, Davids, Button \& Renshaw, 2015). La percepción y la acción son entendidos como procesos inseparables, ya que para actuar se debe percibir y para poder percibir determinadas fuentes de información se debe actuar. Este modelo resta importancia a los procesos conscientes y al conocimiento explícito del deportista, por este motivo propone un proceso de entrenamiento que fomente el aprendizaje implícito y no consciente (Balagué, Torrents, Pol, \& Seirul·lo, 2014).

El proceso de enseñanza-aprendizaje es entendido en términos de relaciones funcionales y adaptativas entre el deportista y su entorno (Renshaw \& Chow, 2018). El aprendizaje emerge como resultado de un proceso autoorganizativo, pues, el jugador a medida que interacciona con la tarea adquiere nuevos patrones de conducta o modifica los que ya tiene, para vencer a los rivales y superar con éxito las normas o constreñimientos presentes en la tarea (Correia, Carvalho, Araújo, Pereira \& Davids, 2018). Según Chow et al., (2015), los constreñimientos pueden ser de tres tipos: (a) los del ambiente (condiciones climatológicas, presencia de espectadores, etc.); (b) los del jugador (nivel de habilidad, características psicológicas, antropométricas, etc.) y (c) los de la tarea (objetivos, reglas, dimensiones del terre- 
no de juego, espacios que deben ocupar los jugadores, sistema de puntuación, prohibición de determinados comportamientos, etc.). El comportamiento finalmente realizado es fruto de la interacción entre los tres tipos de constreñimientos (Balagué, et al., 2014). Por ejemplo, durante la práctica de una tarea de entrenamiento, como puede ser un partido en situación de juego modificada, el entrenador introduce un nuevo constreñimiento: prohibición del bote. Cada equipo en función de sus constreñimientos individuales (capacidad para desmarcarse, capacidad para lanzar a distancia, capacidad para jugar con el pivote, etc.) realizará los comportamientos que les resulten más adecuados para superar al rival. Incluso, la presencia o ausencia de espectadores (constreñimientos ambientales) condicionará las acciones realizadas.

Los constreñimientos introducidos en la tarea, al condicionar los comportamientos disponibles, favorecen la aparición de determinadas habilidades a la vez que deficultan la realización de otras (Balagué, Pol, Torrents, Ric \& Hristovski, 2019). Para la pedagogía no lineal, la manipulación de los constreñimientos de la tarea es la principal herramienta que posee el entrenador en el proceso de ensañanza-aprendizaje, ya que mediante su manipulación puede orientar el proceso autoorganizativo del jugador hacia la consecución de los objetivos propuestos (Chow, et al., 2007).

La tarea a la que se aplican los constreñimientos debe ser representativa del juego real, respetando su esencia y siendo coherente con el ciclo percepción-acción (Renshaw \& Chow, 2018). No procede descomponer el juego y entrenar sus elementos de forma aislada. En lugar de descomponer, los entrenadores deben simplificar (Renshaw, Chow, Davids \& Hammond, 2010). Las tareas se pueden simplificar reduciendo el número de jugadores (compañeros y adversarios), el espacio de juego, el tiempo de juego, las dimensiones del área, etc. Por este motivo, los juegos reducidos encajan perfectamente en el marco teórico de la pedagogía no lineal (Renshaw, et al., 2010), pues aunque presentan diferencias respecto al juego real, las acciones se realizan en contextos similares a los competitivos y no desmontan el ciclo percepción-acción (Serra-Olivares \& García-Rubio, 2017). Este tipo de tareas facilita la transferencia de los aprendizajes y son apropiadas para para jugadores que se encuentran en una fase inicial del aprendizaje, buscando estabilizar posibles soluciones (comportamientos individuales o colectivos), y también, para jugadores que se encuentran en etapas más avanzadas, que buscan optimizar comportamientos ya estabilizados (Renshaw \& Chow, 2018).

Debido a su relevancia, se hace necesario obtener conocimiento empírico que ayude a profundizar en la influencia que ejercen determinados constreñimientos en el proceso de enseñanza-aprendizaje (Renshaw \& Chow, 2018). Con este propósito, en lo últimos años se han realizado diversos estudios centrados en diferentes deportes colectivos como fútbol (Práxedes, et al., 2019; Práxedes, et al., 2018; Roberts, et al., 2019), rugby (Cantos \& Moreno, 2019) y voleibol (Paulo, Infante \& Araujo, 2019). Sin embargo, hasta donde conocemos no existen trabajos que aborden específicamente, desde la pedagogía no lineal, la influencia de los constreñimientos de la tarea en el proceso de enseñanzaaprendizaje en balonmano.
La enseñanza del balonmano se ha estructurado fundamentalmente en torno a los sistemas defensivos: 6:0 (sistema zonal de una línea) y 5:1 (sistema zonal de dos líneas con defensor avanzado) (Román, 2005). Estos sistemas son los más utilizados en balonmano y presentan una diferente manera de ocupar los espacios, expresada en la profundidad (defensores más o menos alejados de la propia portería) y densidad defensiva (defensores más o menos próximos entre sí) (Román, 2015). Esto hace que cada sistema tenga unos puntos fuertes y unos puntos débiles. Son diversos los autores que han señalado los comportamientos, ofensivos y defensivos, más apropiados para los contextos que genera cada sistema (Antón, 2000; Ávila, 2015; Román, 2005; Román 2016).

Por otro lado, a la hora de valorar la eficacia de las propuestas metodológicas empleadas en los procesos de enseñanza-aprendizaje, se han señalado problemas relacionados con los procedimientos empleados para ello. En ocasiones, la evaluación se realiza en contextos muy alejados a los que presenta el juego real (Camacho, 2008). Por este motivo, se deben considerar instrumentos de medida más ecológicos, que respeten la incertidumbre propia de los deportes colectivos, para valorar la realización de determinadas habilidades $\mathrm{y}$, en consecuencia, la eficacia de las propuestas metodológicas empleadas en su enseñanza (Camacho, 2008; Roberts, et al., 2019). Al respecto, la metodología observacional se ha mostrado especialmente válida para estudiar la complejidad inherente a los deportes colectivos, permitiendo un estudio de las conductas realizadas en el contexto real de juego (Anguera \& Hernández-Mendo, 2013). Su utilización es muy frecuente en el estudio del balonmano en alto rendimiento (Flores \& Anguera, 2018; Jiménez-Salas, Morillo-Baro, Reigal, Morales-Sánchez \& Hernández-Mendo, 2020; Lozano, 2014; Lozano, Camerino \& Hileno, 2016; Montoya, 2010; Sousa, Prudente, Sequeira, López-López \& Hernández-Mendo, 2015). En cambio, para el estudio del proceso de enseñanza-aprendizaje no es tan habitual su empleo.

En consecuencia, para la pedagogía no lineal el rol del entrenador no pasa por prescibir las acciones que deben realizar los jugadores, sino más bien por desarrollar las siguientes funciones: (a) diseñar tareas representativas que permitan a los jugadores descubrir sus posibilidades de actuación; (b) conocer la influencia que ejercen los constreñimientos aplicados en las tareas en los comportamientos de los jugadores; (c) conocer cuáles son los constreñimientos de la tarea más adecuados para la adquisición de los objetivos propuestos; y por último, (d) tener presente que el influjo ejercido por un determinado constreñimiento no se expresa por igual en todos los jugadores (Correia, et al., 2018; Renshaw \& Chow, 2018).

Todo esto, sumado a la escasez de investigaciones que aborden la implantación de la pedagogía no lineal en el proceso de enseñanza-aprendizaje en balonmano motivó el presente trabajo; cuyo objetivo fue conocer la influencia que ejercen los constreñimientos: obligatoriedad de utilizar un sistema defensivo zonal de una línea (LI1) y obligatoriedad de utilizar un sistema defensivo zonal de dos líneas con defensor avanzado (LI2), en los comportamientos, ofensivos y defensivos, realizados por jugadores de balonmano durante 
la disputa de partidos en una situación de juego modificado. La obtención de evidencias al respecto, podría ayudar en el diseño de los programas de entrenamiento, evitando que los entrenadores seleccionen los constreñimientos a introducir en las tareas en base a la intuición y al conocimiento subjetivo (Renshaw \& Chow, 2018).

\section{Material y método}

Para la consecución de los objetivos planteados en este trabajo se ha empleado la metodología observacional; en concreto, de acuerdo con Anguera, Blanco-Villaseñor, Hernández-Mendo \& Losada (2011), se ha planteado un diseño observacional: nomotético, porque se estudiaron los comportamientos realizados por dos grupos (naranja y azul); de seguimiento intra-sesional, debido a que los observadores registraron y codificaron las grabaciones de cada una de las sesiones del estudio; puntual, porque los datos obtenidos en las distintas sesiones se analizaron de manera conjunta; y multidimensional, porque se estudiaron diversos niveles de respuesta (comportamientos) que se corresponden con los criterios del instrumento de observación.

\section{Participantes}

En el estudio participaron 14 jugadores de balonmano con una media de edad de 14.6 años y una desviación típica de 0.4. Los participantes fueron todos los integrantes de un equipo de balonmano de categoría cadete masculina, que competían a nivel provincial en la región de Andalucía (España). Los participantes (10 de primer año y cuatro de segundo año) fueron divididos aleatoriamente en dos equipos para disputar 16 partidos, en situación de juego modificado de cuatro contra cuatro más portero, a los que se aplicaron los constreñimientos objeto de estudio. El estudio se desarrolló de acuerdo a los estándares éticos aplicables a la metodología observacional, establecidos en la Declaración de Helsinki. Asimismo, los padres y madres tras ser informados sobre los aspectos esenciales de la investigación, dieron su consentimiento a que sus hijos participaran en el estudio.

\section{Instrumento de observación}

Para registrar los comportamientos de los jugadores en cada una de las sesiones de esta investigación, se construyó un instrumento de observación ad hoc (tabla 1). Debido a la falta de construcciones teóricas y el carácter multidimensional de los comportamientos a estudiar, se optó un diseñó que combinó el formato de campo con sistemas de categorías exhaustivas y mutuamente excluyentes. Esta combinación permite aprovechar los puntos fuertes de ambos componentes, por un lado, el sistema de categorías ofrece consistencia teórica, mientras que, el formato de campo aporta flexibilidad a la hora de registrar los comportamientos específicos que se van a estudiar en la investigación (Anguera, Magnusson \& Jonsson, 2007).

El propósito fue confeccionar un instrumento que permitiese registrar los comportamientos más relevantes en las fases de ataque y defensa posicional, prestando especial atención al juego con el pivote. La construcción del instrumento se desarrolló en tres fases. En un primer momento, se construyó una versión inicial a partir de la revisión teórica
Tabla 1.

\begin{tabular}{|c|c|c|}
\hline Criterio & Categoría y código & Descripción \\
\hline \multirow{3}{*}{ Constreñimiento } & Sistema defensivo de una & El partido se juega con la obligatoriedad para ambos \\
\hline & $\begin{array}{l}\text { línea (LI1) } \\
\text { Sistema defensivo de dos }\end{array}$ & $\begin{array}{l}\text { equipos de utilizar un sistema defensivo de una línea } \\
\text { El partido se juega con la obligatoriedad para ambos }\end{array}$ \\
\hline & $\begin{array}{l}\text { líneas con defensor } \\
\text { avanzado (LI2) }\end{array}$ & $\begin{array}{l}\text { equipos de utilizar un sistema defensivo de dos } \\
\text { líneas con defensor avanzado }\end{array}$ \\
\hline \multirow{2}{*}{ Equipo } & Equipo naranja (NAR) & Ataca el equipo naranja \\
\hline & Equipo azul (AZU) & Ataca el equipo azul \\
\hline \multirow{7}{*}{ Número de pases } & Ningún pase ( $\mathrm{P} 00)$ & No se realiza ningún pase en el ataque \\
\hline & Un pase (P01) & Se realiza un pase en el ataque \\
\hline & Dos pases ( $\mathrm{P} 02)$ & Se realizan dos pases en el ataque \\
\hline & $\mathrm{s}$ Tres pases ( $\mathrm{P} 03$ ) & Se realizan tres pases en el ataque \\
\hline & Cuatro pases (P04) & Se realizan cuatro pases en el ataque \\
\hline & Cinco pases (P05) & Se realizan cinco pases en el ataque \\
\hline & Seis pases ( $\mathrm{P} 06)$ & Se realizan seis o más pases en el ataque \\
\hline \multirow{5}{*}{ Número de botes } & Ningún Bote (B00) & Ningún bote se realiza durante el ataque \\
\hline & Un bote (B01) & Se bota una vez durante el ataque \\
\hline & $\mathrm{s}$ Dos botes (B02) & Se realizan dos botes durante el ataque \\
\hline & Tres botes (B03) & Se realizan tres botes durante el ataque \\
\hline & Cuatro o más botes (B04 & Se realizan cuatro o más botes durante el ataque \\
\hline \multirow{6}{*}{$\begin{array}{l}\text { Medios tácticos } \\
\text { básicos para } \\
\text { iniciar el ataque }\end{array}$} & Fijación impar (ASI) & $\begin{array}{l}\text { El primer medio táctico básico que se realiza en el } \\
\text { ataque es una fijación impar }\end{array}$ \\
\hline & Pase y va (PYV) & $\begin{array}{l}\text { El primer medio táctico básico que se realiza en el } \\
\text { ataque es un pase y va }\end{array}$ \\
\hline & Cruce (CRU) & $\begin{array}{l}\text { El primer medio táctico básico que se realiza en el } \\
\text { ataque es un cruce }\end{array}$ \\
\hline & Permuta (PMT) & $\begin{array}{l}\text { El primer medio táctico básico que se realiza en el } \\
\text { ataque es una permuta }\end{array}$ \\
\hline & $\begin{array}{l}\text { Dos contra dos con } \\
\text { pivote (PVT) }\end{array}$ & $\begin{array}{l}\text { El primer medio táctico básico que se realiza en el } \\
\text { ataque es un dos contra dos con pivote }\end{array}$ \\
\hline & Otro (OTR) & $\begin{array}{l}\text { Se realiza otro medio táctico básico diferente para } \\
\text { iniciar el ataque }\end{array}$ \\
\hline \multirow{5}{*}{ Acción del pivote } & Bloqueo (BLO) & $\begin{array}{l}\text { La primera acción que realiza el pivote es un } \\
\text { bloqueo }\end{array}$ \\
\hline & Desmarque (DES) & La primera acción que realiza el pivote es un \\
\hline & & desmarque \\
\hline & Sale a recibir (JEL) & $\begin{array}{l}\text { La primera acción que realiza el pivote es abandonar } \\
\text { la primera línea defensiva para pedir el balón }\end{array}$ \\
\hline & Otra (OTD) & El pivote realiza otra acción \\
\hline \multirow{4}{*}{$\begin{array}{l}\text { Medios tácticos } \\
\text { básicos } \\
\text { defensivos }\end{array}$} & Doblaje (DBL) & $\begin{array}{l}\text { El doblaje es el medio táctico básico empleado para } \\
\text { defender las acciones donde no está implicado el } \\
\text { pivote }\end{array}$ \\
\hline & $\begin{array}{l}\text { Cambio de oponente } \\
\text { (CMB) }\end{array}$ & $\begin{array}{l}\text { El cambio de oponente es el medio táctico básico } \\
\text { empleado para defender las acciones donde no está } \\
\text { implicado el pivote }\end{array}$ \\
\hline & Deslizamiento (DLZ) & $\begin{array}{l}\text { El deslizamiento es el medio táctico básico } \\
\text { empleado para defender las acciones donde no está }\end{array}$ \\
\hline & Otro (OTD) & Se emplea otro medio táctico básico \\
\hline \multirow{3}{*}{$\begin{array}{l}\text { Defensa del } \\
\text { pivote }\end{array}$} & Contrabloqueo $(\mathrm{CON})$ & $\begin{array}{l}\text { El contrabloqueo es el medio táctico básico } \\
\text { empleado para defender el juego con el pivote }\end{array}$ \\
\hline & Deslizamiento (DZM) & $\begin{array}{l}\text { El deslizamiento es el medio táctico básico } \\
\text { empleado para defender el juego con el pivote }\end{array}$ \\
\hline & Otro (OTI) & $\begin{array}{l}\text { Se realizan otros medios tácticos para defender el } \\
\text { juego con el pivote }\end{array}$ \\
\hline \multirow{5}{*}{$\begin{array}{l}\text { Tipo de } \\
\text { lanzamiento }\end{array}$} & Finta (FTA) & $\begin{array}{l}\text { El ataque finaliza con lanzamiento después de una } \\
\text { finta }\end{array}$ \\
\hline & $\begin{array}{l}\text { Lanzamiento a distancia } \\
\text { (LLJ) }\end{array}$ & El ataque finaliza con un lanzamiento a distancia \\
\hline & Penetración (PEN) & $\begin{array}{l}\text { El ataque finaliza con lanzamiento después de una } \\
\text { penetración }\end{array}$ \\
\hline & $\begin{array}{l}\text { Lanzamiento de pivote } \\
\text { (PVO) }\end{array}$ & $\begin{array}{l}\text { El ataque finaliza con un lanzamiento desde el } \\
\text { pivote }\end{array}$ \\
\hline & Otro (OTI) & $\begin{array}{l}\text { El ataque finaliza con un lanzamiento después de } \\
\text { realizar otra acción. }\end{array}$ \\
\hline \multirow{5}{*}{$\begin{array}{l}\text { Acción defensiva } \\
\text { final }\end{array}$} & Uno contra uno (UX1) & $\begin{array}{l}\text { La acción individual defensiva realizada en el } \\
\text { momento de la finalización del ataque fue un uno } \\
\text { contra uno }\end{array}$ \\
\hline & Blocaje (BLC) & $\begin{array}{l}\text { La acción individual defensiva realizada en el } \\
\text { momento de la finalización del ataque fue un blocaje }\end{array}$ \\
\hline & Robo en bote (RBT) & $\begin{array}{l}\text { La acción individual defensiva realizada en el } \\
\text { momento de la finalización del ataque fue un robo } \\
\text { en bote }\end{array}$ \\
\hline & Interceptación (ITR) & $\begin{array}{l}\text { La acción individual defensiva realizada en el } \\
\text { momento de la finalización del ataque fue una } \\
\text { interceptación }\end{array}$ \\
\hline & Marcaje al pivote (MPV & $\begin{array}{l}\text { La acción individual defensiva realizada en el } \\
\text { momento de la finalización del ataque fue para } \\
\text { detener al pivote }\end{array}$ \\
\hline \multirow{3}{*}{$\begin{array}{l}\text { Finalización del } \\
\text { ataque }\end{array}$} & Lanzamiento (LAN) & El ataque finaliza con un lanzamiento \\
\hline & Pérdida de balón (PER) & El ataque finaliza con una pérdida de balón \\
\hline & 1 Interrupción (INT) & $\begin{array}{l}\text { El ataque finaliza con señalización de golpe franco o } \\
\text { saque de banda, sin que se pierda la posesión de }\end{array}$ \\
\hline \multirow{4}{*}{$\begin{array}{l}\text { Zona de } \\
\text { finalización }\end{array}$} & Zona izquierda (ZIZ) & El ataque finaliza en la zona izquierda (figura 1 ) \\
\hline & Zona derecha (ZDE) & El ataque finaliza en la zona derecha (figura 1) \\
\hline & Zona central (ZCN) & El ataque finaliza en la zona central (figura 1) \\
\hline & Campo propio (CPR) & El ataque finaliza en el campo propio (figura 1) \\
\hline
\end{tabular}

efectuada, siendo de especial utilidad el trabajo de Lozano et ál. (2016). Posteriormente, se registraron tres partidos, de similares características a los que formaron parte del estudio, que no fueron incluidos en la muestra. Al no detectarse nuevas conductas en ningún criterio, se dio por superada la prueba de cautela (Anguera, et al., 2007). Por último, el instrumento fue sometido a un juicio de expertos, en el que participaron cinco personas. Todos entrenadores naciona- 
les de balonmano: dos eran entrenadores de la selección andaluza de balonmano y otros dos profesores de la asignatura de balonmano en el grado de ciencias de la actividad física y del deporte. Se siguió un procedimiento similar al realizado en los trabajos de Lozano (2014) y Montoya (2010). En un primer momento se procedió a la lectura y explicación de todos los criterios y categorías del instrumento de observación. Posteriormente, los expertos rellenaron una plantilla en la que debían marcar su acuerdo o desacuerdo con cada uno de ellos. Finalmente, cada criterio y categoría del instrumento de observación obtuvo un porcentaje de acuerdo igual o superior al $80 \%$.

El juicio de los expertos sirvió para reforzar la validez del instrumento de observación, apoyada también en la descripción de los criterios y categorías que lo conforman, en el marco teórico utilizado para su elaboración (Anguera, 2003; Prudente, Garganta \& Anguera, 2004) y en la realización del análisis de generalizabilidad. Finalmente, el instrumento de observación quedó conformado por 12 criterios y 50 categorías. Se registraron y codificaron cada una de las posesiones de ambos equipos. De manera que las unidades de observación se iniciaban cuando un equipo recuperaba el balón y finalizaban cuando: a) perdían la posesión del balón después de realizar un lanzamiento o cometer un error técnico o reglamentario; b) se daba una interrupción del juego, como por ejemplo un golpe franco o saque de banda, sin que esta interrupción acarrease un cambio en la posesión del balón.

\section{Instrumentos de registro}

La grabación de los partidos fue realizada con una cámara Sony FDR-AX33 situada a 4 metros de altura detrás de una de las porterías. Posteriormente, para el registro y codificación de las acciones se introdujo el instrumento de observación en el programa informático Dartfish 5.5. El análisis de la calidad de dato se realizó utilizando el programas informáticos GSEQ 5.1 (Bakeman \& Quera, 2011) para el cálculo de la concordancia intra e interobservadores; y EduG versión 6.1 (Cardinet, Johnson \& Pini, 2010) para realizar el análisis generalizabilidad, que permitió conocer la homogeneidad de las categorías, determinar la fiabilidad de los observadores y el grado en el que se pueden generalizar los resultados obtenidos con precisión. Finalmente, para conocer los patrones de conducta realizados por los jugadores en presencia de los constreñimientos aplicados, se aplicó el análisis de las coordenadas polares empleando el programa informático HOISAN 1.2 (Hernández-Mendo, López, Castellano, Morales \& Pastrana, 2012). Anteriormente, como requisito previo al cálculo de coordenadas polares, se realizó el análisis secuencial de retardos utilizando de nuevo el programa informático GSEQ 5.1. Se consideraron retardos de +1 $\mathrm{a}+5$ para la perspectiva prospectiva y de -1 a -5 para la retrospectiva. Una vez conocidas las relaciones significativas, detectadas por el análisis de coordenadas polares, se representaron gráficamente por medio del programa informático Snowflake 0.2.

\section{Procedimiento}

El estudio se desarrolló a lo largo de ocho sesiones que se desarrollaron en lugar y horario habitual de entrenamiento. Todas las sesiones tuvieron la misma estructura: un ca- lentamiento (de 10 minutos de duración), seguido de una parte principal (40 minutos); y por último una tarea de vuelta a la calma (10 minutos). En la parte principal de cada sesión se jugaron dos partidos en una situación de juego modificado de 4 contra 4 más portero, que estuvieron condicionados por la introducción de los constreñimientos objeto de estudio (figura 1): (1) obligatoriedad de utilizar un sistema defensivo zonal de una línea (LI1), y (2) obligatoriedad de utilizar un sistema defensivo zonal de dos líneas con defensor avanzado (LI2). Estos constreñimientos son representativos de los dos sistemas defensivos más utilizados en balonmano: el sistema 6:0, considerado un sistema defensivo cerrado, y el sistema defensivo 5:1, catalogado como abierto (JiménezSalas, et al. 2020).

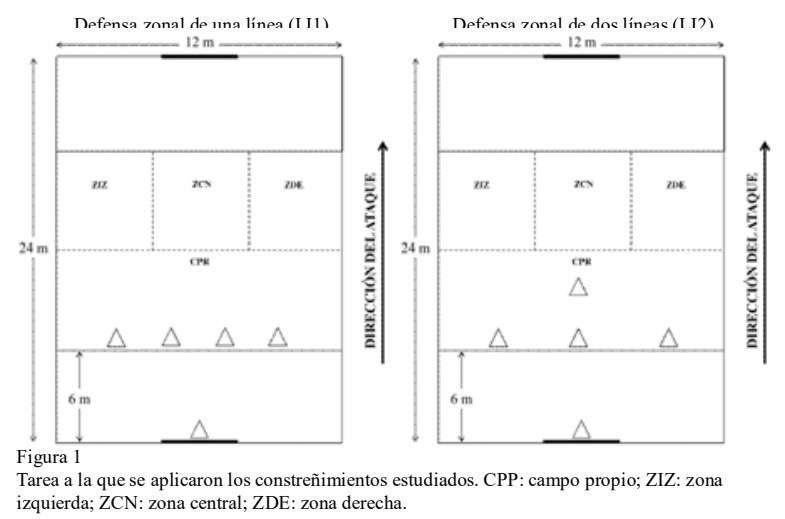

En cada sesión se disputaron dos partidos, uno en presencia de cada constreñimiento estudiado. El orden de aparición de los constreñimientos se fue alternando a lo largo de las distintas sesiones, empezando por el constreñimiento defensa zonal de una línea(LI1). Los partidos se jugaron con las reglas oficiales de balonmano exceptuando a los saques después de gol, que los realizó el portero dentro de su área; y los saques de golpe franco, que se efectuaron desde el punto central del campo. Además, como el propósito de este estudio fue conocer la influencia ejercida por dos constreñimientos representativos de los sistemas defensivos zonales, se prohibieron los contraataques y en todo momento, los equipos jugaron en igualdad numérica. La elección de este tipo de tareas (partido en situación modificada) para la consecución de los objetivos del estudio se justifica porque al ser representativas del deporte real, conservan su estructura y esencia, permite que los jugadores realicen las habilidades individuales y de interacción en contextos próximos a los del juego real (Chow, et al., 2015). De manera que, las tareas representativas y que respetan la naturaleza del ciclo percepción-acción encajan perfectamente en el marco de la pedagogía no lineal, siendo adecuada su utilización tanto en niveles iniciales del aprendizaje, como en niveles más avanzados (Renshaw \& Chow, 2018). Para la disputa de los partidos, los 14 participantes fueron asignados a dos equipos que no se modificaron a lo largo del estudio. De esta manera, la influencia de los constreñimientos individuales se controló, al menos en parte, y se pudieron conocer las tendencias de comportamiento que presentó cada equipo según el constreñimiento aplicado. La composición de los equipos se realizó aleatoriamente, pero con la intención de que los equipos fueran homogéneos se estableció el siguiente 
criterio: ambos equipos debían tener, como mínimo, un jugador especialista en las posiciones de portero, pivote y primera línea. Cada partido tuvo una duración de ocho minutos y hubo un periodo de cinco minutos de descanso entre partidos. Cada equipo tomó las decisiones que creó oportunas respecto a las alineaciones y sustituciones realizadas durante el partido. Asimismo, ni antes, ni durante, ni después de los partidos, el entrenador proporcionó ningún tipo de indicación relacionada con los comportamientos a realizar.

\section{Calidad de dato}

El registro y la codificación de las acciones fue realizada por dos observadores, ambos entrenadores nacionales de balonmano y con experiencia en metodología observacional. Para optimizar la fiabilidad de las observaciones, participaron en un proceso de entrenamiento que tuvo dos fases. La primera, como ambos tenían conocimientos sobre los conceptos básicos de la metodología observacional, se centró específicamente en la comprensión de cada uno de los criterios y categorías del instrumento de observación. Para ello se utilizó la grabación de partidos similares a los que posteriormente formaron parte de la muestra, de manera que los observadores pudieron discutir a qué categoría pertenecían las acciones observadas. En la segunda fase del entrenamiento se registraron y codificaron distintos partidos que no fueron incluidos en la muestra. El proceso de entrenamiento concluyó cuando se obtuvieron niveles de concordancia superiores a .80 en el estadístico Kappa de Cohen para todos los criterios, tanto a nivel intraobservador, una misma sesión registrada por el mismo observador en dos momentos diferentes, como a nivel interobservadores, una misma sesión registrada por los dos observadores.

Una vez finalizada la fase de entrenamiento, se procedió al registro y codificación de los comportamientos realizados en cada uno de los partidos disputados en el estudio. Se calcularon los niveles de concordancia intraobservador e interobservadores, obteniéndose en ambos casos un índice Kappa de Cohen superior a .92 en todos los criterios. El nivel de acuerdo mostrado puede considerarse casi perfecto (Landis \& Koch, 1977).

Para valorar la homogeneidad de las categorías, determinar la fiabilidad de los observadores y el grado en el que se pueden generalizar los resultados obtenidos con precisión, se realizó el análisis de generalizabilidad (Cronbach, Gleser, Nanda \& Rajaratnam, 1972). Para conocer la homogeneidad de las categorías se planteó un diseño de dos facetas observadores / categoría $(\mathrm{O} / \mathrm{C})$, obteniéndose unos coeficientes de generalización, relativo y absoluto, igual a cero. Estos resultados indican que las categorías son adecuadas y cumplen con el requisito de ser exhaustivas y mutuamente excluyentes, por lo que se puede asumir que la homogeneidad de las categorías es óptima. Para determinar la fiabilidad de las observaciones a nivel interobservadores, se empleó un diseño de dos facetas categoría / observadores $(\mathrm{C} / \mathrm{O})$, obteniéndose coeficientes de generalización, tanto absolutos como relativos, por valor de .99 en ambos niveles de análisis. Asimismo, el análisis de la varianza indicó que el $98.2 \%$ de la variabilidad encontrada queda asociada a la faceta categorías, lo que supone un excelente resultado. El mismo diseño se empleó para estudiar la fiabilidad intraobservador, obteniéndose coeficientes de generalización absoluto y relativo igual a uno y un $99.3 \%$ a de la variabilidad encontrada asociada a la faceta categorías, suponiendo también unos excelentes resultados. Para finalizar con el análisis de generalizabilidad y poder estimar el grado en el que se pueden generalizar los resultados obtenidos tras registrar 16 partidos, se tomó un diseño de dos facetas categorías / sesiones $(\mathrm{C} / \mathrm{S})$. Los índices de generalizabilidad obtenidos a partir de la observación de 16 partidos fueron de .94467 para el coeficiente G relativo y de .94055 para el coeficiente $\mathrm{G}$ absoluto. Estos valores indicaban que con la observación de 16 partidos se obtiene un excelente grado de generalización de los resultados.

\section{Análisis de coordenadas polares}

En los últimos años, el análisis de coordenadas ha sido empleado en importantes investigaciones en el ámbito de los deportes colectivos (Castañer, Barreira, Camerino, Anguera, Canton \& Hileno, 2016; Castañer, Barreira, Camerino, Anguera, Fernandes \& Hileno, 2017; Prudente, Cardoso, Rodrigues \& Sousa, 2019), mostrándose como un método emergente en el análisis del comportamiento táctico en los deportes de equipo (Ávila-Moreno, Chirosa-Ríos, Ureña-Espá, Lozano-Jarque \& Ulloa-Díaz, 2018).

Su aplicación permite conocer los patrones de conducta que emergen en contextos de mucha incertidumbre, como son los propios del balonmano. En concreto, posibilita la representación gráfica las asociaciones, de activación o inhibición, existentes entre los comportamientos que son objeto de estudio. A los comportamientos estudiados se les asignan dos roles: conducta focal, a la que se considera generadora de las relaciones; y conductas condicionadas, al resto de comportamientos implicados en la asociación.

Para ello, la técnica de coordenadas polares desarrolla un análisis secuencial prospectivo y retrospectivo, con igual número de retardos, que complementa entre sí de acuerdo con la propuesta de Sackett (1980). Para la perspectiva prospectiva, que pretende conocer en qué medida hacia delante existe relación significativa, se consideran los retardos positivos, y para la perspectiva retrospectiva, que permite conocer en qué medida hacia atrás existe relación significativa, se consideran los retardos negativos.

La integración de ambos niveles de análisis se realiza mediante la aplicación del estadístico $Z_{\text {sum }}=\frac{\sum Z}{\sqrt{n}}$ (Sackett, 1980). Este estadístico es considerado una potente técnica de reducción de datos y se aplica tanto a la vertiente prospectiva como a la retrospectiva. Cada Zsum prospectivo y retrospectivo puede tener signo positivo o negativo. La combinación de los signos y valores prospectivos y retrospectivos, determinará en cuál de los cuatro cuadrantes posibles (I, II, III, IV) se representará gráficamente la asociación (figura 2). El cuadrante I indica una relación de activación mutua entre la conducta focal y la condicionada; el cuadrante IV se indica que la conducta focal activa a la condicionada, mientras que es inhibida por ella; el cuadrante III se señala una relación de inhibición mutua entre ambas conductas; y por último, el cuadrante II implica que la conducta focal inhibe a la condicionada, mientras que la condicionada activa a la focal (Anguera, et al., 2011). 

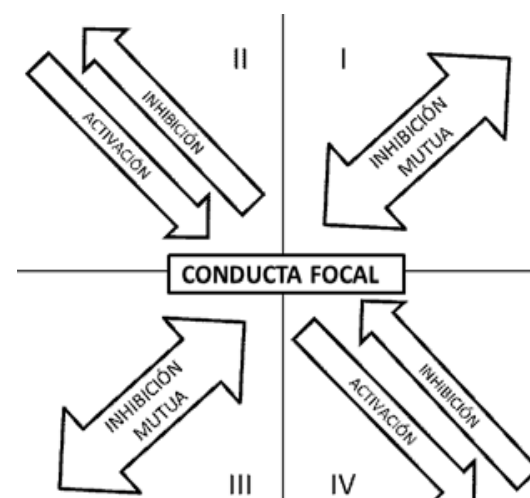

III

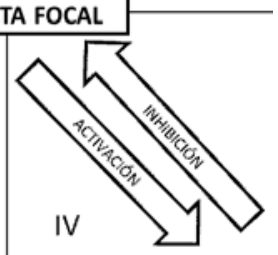

Figura 2

Representación gráfica de las relaciones entre la conducta focal y las condicionadas según cuadrante en el que se ubique la relación (Castañer, et al, 2016).

\section{Resultados}

A continuación, en las figuras $3,4,5,6,7$ y 8 se recogen los resultados significativos $>1.96(p<.05)$, obtenidos tras la aplicación de la técnica de coordenadas polares. Para simplificar la comprensión de los mismos se comentarán las relaciones ubicadas en el cuadrante I, es decir, aquellas que expresan una relación de mutua activación entre la conducta focal y las condicionadas.

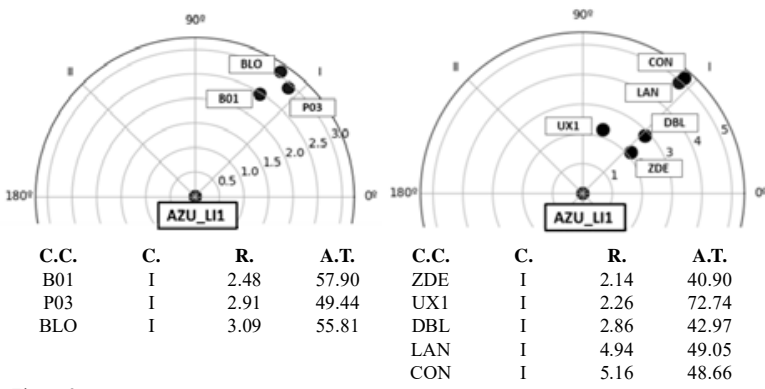

Figura 3 .

. BO1: un bote; P03: tres pases; BLO: bloqueo del pivote; ZDE: zona derecha; UX1: uno contra uno; DBL: doblaje; LAN: lanzamiento; CON: contrabloqueo.

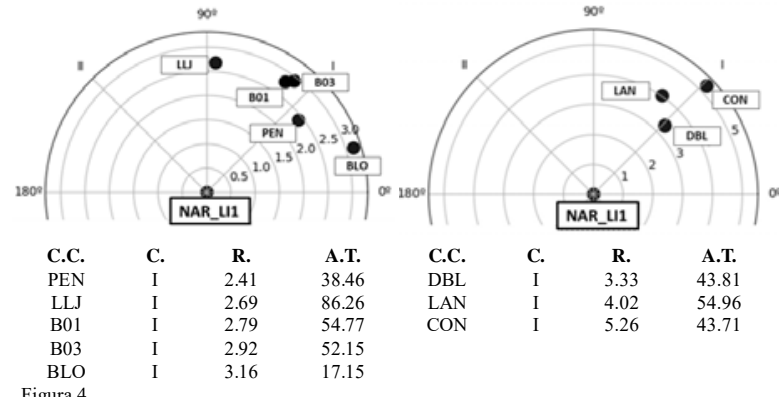

Asociaciones significativas cuando el equipo naranja ataca a un sistema defensivo de una línea. PEN: Penetración; LLJ: Lanzamiento a distancia; B01: un bote; B03: tres botes; BLO: bloque DBL: doblaje; LAN: lanzamiento; CON: contrabloqueo.

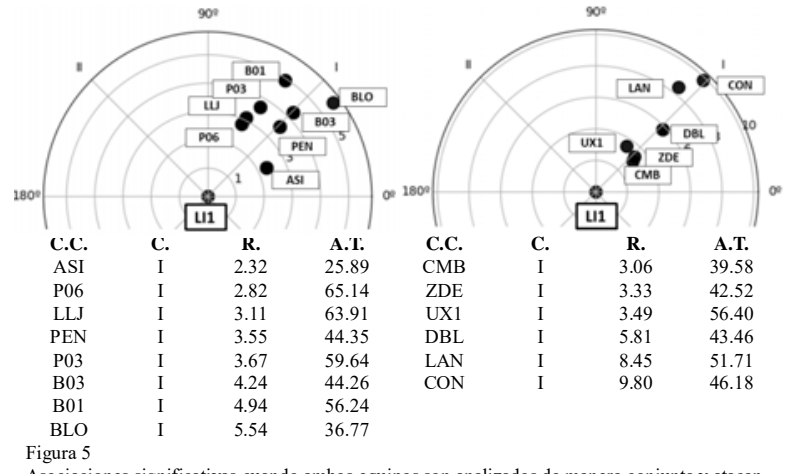

Tignificativas cuando ambos equipos son analizados de maneaconjunta y ataco un sistema defensivo de una línea ASI: fijación impar; P06: seis o más pases; LLJ: lanzamiento a distancia; PEN: penetración; $\mathrm{P} 03$ : tres pases; $\mathrm{B} 03$ : tres botes; B01: un bote; BLO: bloqueo; CMB: cambio de oponente; ZDE: zona derecha; UX1: uno contra uno; DBL: doblaje; LAN: lanzamiento; CON: contrabloqueo.

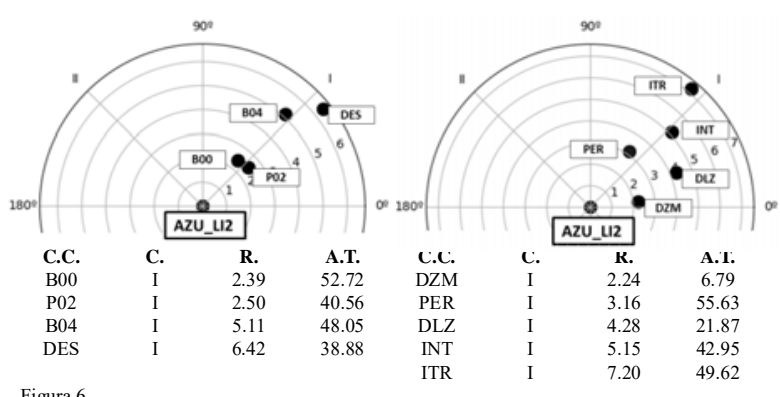

Figura 6

Asociaciones significativas cuando el equipo azul ataca a un sistema defensivo de dos líneas con defensor avanzado. BOO: ningún bote; P02: dos botes, B04: cuatro o más botes; DES: desmarque; PYR: error técnico o reglamentario; DZM: deslizamiento; PER: pérdida; DLZ: deslizamiento para marcar al pivote; INT: interrupción; ITR: interceptación.

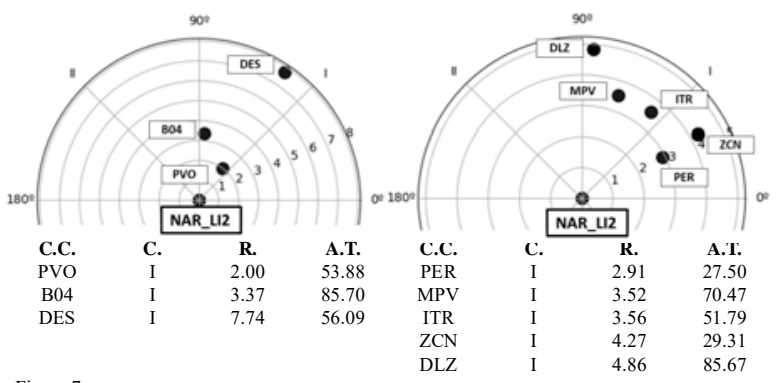

Asociaciones significativas cuando el equipo naranja ataca a un sistema defensivo de dos líneas con defensor avanzado. PVO: acción pivote; PYR: error técnico o reglamentario; B04: cuat ZCN; zona central; DLZ: deslizamiento.

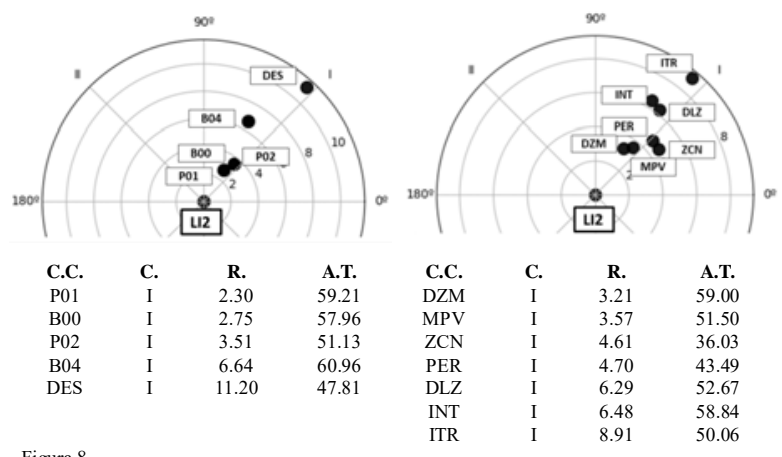

Figura 8

Asociaciones significativas cuando ambos equipos son analizados de manera conjunta y ataca a un sistema defensivo de dos lineas con defensor avanzado. P01: un pase; B00: ningún bote; P02: dos pases; B04: cuatro botes o más; PYR: error técnico o reglamentario; DES: desmarque; DZM: deslizamiento; MPV: marcaje a pivote; ZCN: zona central; PER: pérdida; DLZ: deslizamiento; INT: interrupción; ITR: interceptación.

Como conductas focales actuaron los constreñimientos estudiados, defensa zonal de una línea (LI1) en las figuras 3, 4 y 5 , y defensa zonal en dos líneas con defensor avanzado (LI2) en las figuras 6, 7 y 8. Cada una de las figuras está conformada por dos columnas. La de la izquierda tiene como conductas condicionadas las categorías pertenecientes a los criterios: número de pases, número de botes, medios tácticos básicos para iniciar el ataque, tipo de lanzamiento y acciones del pivote. Por su parte, en la columna de la derecha se emplean las siguientes conductas condicionadas: tipo de lanzamiento, zona de finalización, medios tácticos básicos defensivos, acción defensiva final y defensa del pivote.

\section{Defensa zonal de 1 línea (LI1)}

Al observar la figura 3, en la columna de la izquierda, se representan los ataques del equipo azul contra la defensa zonal de una línea del equipo naranja (AZU_LI1). Los resultados mostraron activación mutua con la realización de un bote $(\mathrm{B} 01)$, la realización de tres pases $(\mathrm{P} 03)$ y la realización de bloqueos por parte del jugador que juega en la posición 
de pivote (BLO). En la columna de la derecha, se puede apreciar relación de activación mutua con la finalización de ataque mediante lanzamientos (LAN), finalización de los ataques en la zona de la derecha (ZDE) y con la realización en defensa de: unos contra uno (UX1), doblajes (DBL) y contrabloqueos para defender el juego con pivote (CON).

En la figura 4, actúa como conducta focal los ataques realizados por el equipo naranja ante un sistema defensivo zonal de una línea (NAR_LI1). En la columna de la izquierda vemos activación mutua con ataques donde se realiza un bote (B01), ataques donde se realizan tres botes (B03), ataques que finalizan después de una penetración (PEN), ataques que finalizan con un lanzamiento a distancia (LLJ) y ataques donde el pivote realiza bloqueos (BLO). Asimismo, en la columna de la derecha podemos ver relación de activación mutua con la finalización de los ataques mediante lanzamientos (LAN) y la realización en defensa de doblajes (DBL) y contrabloqueos para defender el juego con el pivote (CON).

$\mathrm{Al}$ analizar los ataques de ambos equipos de manera conjunta (LI1) (figura 5), se observa activación mutua con la realización de un bote (B01), tres botes (B03), tres pases (P03), siete o más pases (P7M), bloqueos del pivote (PVT), fijación impar (ASI), penetraciones para finalizar (PEN) y realización de lanzamientos a distancia (LLJ). Además, en la columna de la derecha se observa relación de activación mutua con la finalización mediante lanzamientos (LAN), en la zona derecha (ZDE) y con la realización en defensa de: cambios de oponente (CMB), doblajes (DBL), contrabloqueos para defender el juego con el pivote (CON) y unos contra uno(UX1).

\section{(LI2)}

Defensa zonal de dos líneas con defensor avanzado

Al observar la figura 6, en la columna de la izquierda, se puede identificar los ataques del equipo azul contra la defensa zonal de dos líneas con defensor avanzado (AZU LI2). Los resultados obtenidos mostraron activación mutua con la realización de cuatro o más botes (B04), la no utilización del bote (B00), la realización de un pase (P01), la realización de dos pases (P02) y la realización de desmarques por parte del jugador que juega en la posición de pivote (DES). En la columna de la derecha, se puede apreciar relación de activación mutua con la finalización en pérdidas de balón (PER), ataques que finalizan en interrupción (INT), la realización de interceptaciones en defensa (ITR), y la colaboración defensiva por medio de deslizamiento (DLZ), también para defender el juego con pivote (DZM).

En la figura 7, actúa como conducta focal los ataques realizados por el equipo naranja ante un sistema defensivo zonal de dos líneas con defensor avanzado (NAR_LI2). En la columna de la izquierda, vemos activación mutua con ataques donde se realizan cuatro o más botes (B04), ataques que finalizan con una lanzamiento del pivote (PVO) y ataques donde el pivote realiza desmarques (DES). En la columna de la derecha, se puede apreciar relación de activación mutua con la finalización en pérdidas de balón (PER), la realización de interceptaciones en defensa (ITR), la realización de marcaje al pivote en el momento de finalizar el ataque (MPV) y la colaboración defensiva por medio de deslizamiento (DLZ), también para defender el juego con pivote
(DZM).

Para finalizar, al analizar los ataques de ambos equipos conjuntamente (LI2) (figura 8), se observa activación mutua con la realización de cuatro o más botes (B04), de ataques donde no se emplea el bote (B00), ataques donde se realiza un pase (P01), ataques donde se realizan dos pases (P02) y la utilización de desmarques del pivote (DES). Además, en la columna de la derecha se observa relación de activación mutua con la finalización de ataques en pérdida (PER), en interrupción (INT), en la zona central (ZCN) y con la realización en defensa de: deslizamientos, estuviera implicado (DZM) o no el pivote (DLZ), y marcaje al pivote (MPV).

\section{Discusión}

El objetivo de este estudio fue comprobar la influencia ejercida por los constreñimientos LI1 (obligatoriedad de emplear una defensa zonal de una línea) y LI2 (obligatoriedad de utilizar una defensa zonal de dos líneas con defensor avanzado) en los comportamientos realizados por jugadores de balonmano durante la disputa de partidos en una situación de juego modificada. El análisis de coordenadas polares mostró la emergencia de diferentes patrones de conducta, tanto en ataque como en defensa, según el constreñimiento presente.

Para la pedagogía no lineal es clave que el entrenador conozca la influencia que ejercen los constreñimientos introducido en las tareas en los comportamientos de los jugadores (Renshaw \& Chow, 2018). Los constreñimientos analizados en este estudio son representativos de los sistemas defensivos más utilizados en balonmano, el sistema 6:0 (defensa zonal de una línea) y el 5:1(defensa zonal de dos líneas con jugador avanzado). Cada sistema defensivo ofrece diferente profundidad y densidad defensiva. Estas características invitan a la realización de unos comportamientos y, también, dificultan la realización de otros (Román, 2015; Sousa, et al., 2015). Los resultados encontrados en el presente estudio así lo reflejan.

Para atacar sistemas defensivos considerados cerrados, aquellos que tienen una única línea defensiva, las principales herramientas son: el lanzamiento a distancia (Lozano, et al., 2016; Román, 2005) y las penetraciones para aprovechar el espacio entre el defensor lateral y el exterior (Jiménez-Salas, et al., 2020; Román, 2005). Los resultados encontrados en el presente estudio coinciden, pues el constreñimiento defensa zonal de una línea activó los lanzamientos lejanos y los realizados después de penetración.

Respecto al inicio de los ataques, Román (2005) destaca la idoneidad de utilizar medios tácticos que involucren a dos jugadores (dos contra dos con pivote, cruces, permutas, penetraciones sucesivas tras fijar al impar) para generar desequilibrios en la defensa. Asimismo, Jiménez-Salas, et al. (2020) defienden el predominio de cruces y permutas para atacar sistemas defensivos cerrados. Al respecto, en la presente investigación solo se activó la realización de fijaciones al impar para iniciar los ataques contra las defensas de una línea. Quizás, los jugadores de categoría cadete que han participado en este estudio, en su mayoría de primer año, aún no dominan los medios tácticos básicos que citan estos estudios precedentes. 
En cuanto a las acciones del pivote, Sousa et al. (2015) encontraron que la defensa de una línea facilitaba las situaciones de dos contra dos, que involucran al pivote con un jugador de primera línea. Sin embargo, en nuestro estudio no se encontró activación de esta colaboración, aunque sí se activó la realización de bloqueos por parte del pivote. Este dato hay que subrayarlo, pues la importancia del bloqueo ante sistemas defensivos cerrados ya ha sido destacada con anterioridad (Jiménez-Salas, et al., 2020; Román, 2005; Sousa, et al., 2015). Más específicamente, según Román (2005), el pivote puede realizar bloqueos en beneficio propio (para recibir y lanzar) o en beneficio de sus compañeros (para facilitar penetraciones). Los resultados obtenidos coinciden en parte con este autor, pues se constató la activación del bloqueo, pero no la activación de lanzamientos del pivote. Esto indica que el pivote no realizó bloqueos en beneficio propio y sí en beneficio de sus compañeros. Asimismo, Antón (2000) y Román (2005) destacan la importancia de otras acciones del pivote como pantallas y cortinas para facilitar los lanzamientos a distancia de los compañeros; sin embargo, estos comportamientos no se han activado en este estudio. Probablemente porque estos comportamientos aún no forman parte del repertorio conductual de los jugadores en formación que han participado en este estudio.

Por otro lado, los sistemas defensivos de dos líneas con jugador avanzado ofrecen más profundidad, pero tienen menos jugadores en la línea defensiva más próxima a su portería. Este es, a priori, uno de sus puntos débiles; por este motivo el ataque debe tener entre sus principales objetivos finalizar desde la línea de seis metros, ya sea jugando por los extremos o desde el pivote (Román, 2005). En nuestro estudio se han encontrado finalizaciones del pivote solo en el equipo naranja. Esto subraya la importancia de los constreñimientos individuales a la hora de aprovechar las oportunidades que ofrece el juego (Balagué, et al., 2014). Es decir, una misma situación puede ser percibida por un equipo como favorable para jugar con el pivote, mientras que para otro equipo no. Asimismo, Flores y Anguera (2018) demostraron como la presencia de un determinado jugador afecta a los comportamientos realizados por el resto. Por este motivo, la composición de los grupos también pudo influir en los resultados encontrados, en relación a este y a otros comportamientos. Por otro lado, las características del espacio de juego utilizado, la forma recta del área y la distribución de los jugadores en el mismo (no hay jugadores que ocupen los extremos), no permiten que se realicen comportamientos que impliquen a los extremos. Respecto a los movimientos para iniciar el ataque, Lozano et al., (2016) encontraron que los medios tácticos de dos jugadores son los más utilizados para atacar a estos sistemas defensivos, sin precisar exactamente cuáles. En cambio, Román (2005) señaló la utilidad de iniciar los ataques por medio de cruces y permutas para generar problemas al defensor avanzado. Los resultados encontrados en nuestro estudio no van en esta línea, pues no se activó la realización de ningún medio básico para iniciar los ataques. Esto indica que el desarrollo del juego se hizo de manera más directa, más individual, como refleja la activación de ataques donde se realizan menos pases y más botes, en comparación con los realizados frente al sistema defensivo de una línea. La explicación de estos resultados puede estar relacionada, por un lado, con el buen hacer de la defensa de dos líneas, que consigue uno de sus principales objetivos: dificultar el desarrollo del ataque (Lozano, et al., 2016); $y$, por otro, al igual que ocurrió ante la defensa de una línea, puede ser que los jugadores aún no tengan demasiadas herramientas colectivas.

Respecto a las acciones del pivote, Jiménez-Salas et al. (2020) encontraron que los pivotes para atacar a los sistemas defensivos de dos líneas, realizaban los siguientes comportamientos: bloqueo, pantallas, cortinas y pase y va. La utilización del pase y va de manera coordinada con otros medios tácticos también fue destacada por Román (2005). Los jugadores que ocuparon la posición de pivote activaron la realización de desmarques para aprovechar los espacios libres de la primera línea defensiva, uno de los puntos débiles de este sistema (Román 2005). Por otro lado, al igual que encontraron Sousa et al. (2015) la defensa de dos líneas no facilitó la realización de dos contra dos, entre jugadores de la primera línea y pivote. Esta colaboración tampoco se activó ante el sistema defensivo de una línea, esto puede indicar que este comportamiento no es el más apropiado para los jugadores estudiados, con independencia del sistema defensivo que utilice el equipo rival.

En referencia a los comportamientos defensivos, tradicionalmente el sistema defensivo de una línea suele proponer comportamientos reactivos, orientados a evitar o dificultar los lanzamientos del equipo rival. Para ello las principales herramientas son: el blocaje y el acoso, situación donde un atacante y defensor se encuentran en proximidad (Román, 2016). En cambio, el sistema de dos líneas tiene un carácter más activo. Lozano et al. (2016) encontraron que los equipos suelen utilizar este sistema cuando van perdiendo, con el objetivo de recuperar la posesión del balón y de interrumpir el juego de ataque. Los resultados encontrados coinciden plenamente, pues la defensa de una línea activó la finalización de los ataques con lanzamiento y la defensa de dos líneas activó la realización de interceptaciones y la finalización de ataques en interrupciones. En consonancia con su orientación reactiva, la defensa de una línea facilitó la realización de situaciones de uno contra uno pero no activó la realización de blocajes, quizás porque este recurso no es dominado por los jugadores de categoría cadete.

Respecto a los comportamientos colectivos, según Antón (2000) el cambio de oponente es el medio básico fundamental en las defensas zonales, ya que de esta manera cada jugador mantiene su zona de responsabilidad. Los resultados encontrados para la defensa zonal de una línea coinciden con esta idea, sin embargo la defensa zonal de dos líneas activó la realización de deslizamientos. Según Antón (2000), el deslizamiento debe utilizarse de manera esporádica en los sistemas defensivos zonales, utilizándose exclusivamente cuando los defensores estén escalonados (diferente profundidad). La mayor densidad defensiva del sistema de una línea pudo facilitar el cambio de oponente, en cambio, los escalonamientos entre defensores, más frecuentes en la defensa de dos líneas, explicarían la utilización de deslizamientos. La utilización del deslizamiento, según Ávila (2015), incrementa los espacios entre defensores y rompe la cohesión del sistema zonal, individualizando la defensa. Los hallazgos encontrados van en esta línea, pues cuando se acti- 
vó la realización de deslizamientos, en el sistema de dos líneas, se activó el marcaje individual a pivote y no se activó la realización de doblajes (ayuda ante el error). La activación de los doblajes coincidió con la activación de los cambios de oponente, esto ocurría en el caso del sistema defensivo de una línea, que a priori ofrece menos espacios entre defensores. Por otro lado, para defender el juego con pivote, la defensa de una línea propició la colaboración mediante el contrabloqueo, mientras que el sistema zonal de dos líneas favoreció el deslizamiento. La explicación puede encontrarse en el escalonamiento defensivo, más propios de la defensa de dos líneas, que dificulta el contrabloqueo (medio táctico básico que también implica que los defensores cambien de oponente) y facilita el deslizamiento.

En síntesis, es posible afirmar que el entrenador debe conocer la influencia que ejercen los constreñimientos que introduce en sus tareas: qué comportamientos facilita y cuáles inhibe (Renshaw \& Chow, 2018). De esta manera podrá seleccionar los más adecuados, aquellos que guíen a los jugadores hacia la consecución de los objetivos propuestos (Chow, et al., 2007; Correia, et al., 2018;). Los resultados obtenidos aportan información al respecto. Se ha podido encontrar la influencia general que ejercen los constreñimientos estudiados, expresada en tendencias generales de comportamiento para ambos equipos, pero también se han encontrado comportamientos específicos para cada equipo. Esto supone un desafío para los entrenadores, pues deben conocer específicamente para cada jugador o grupo de jugadores las autoorganizaciones que provocan los constreñimientos empleados. Además, hay que subrayar que la técnica de coordenadas polares se ha mostrado como una herramienta útil para informar sobre este proceso, clave para la implantación de la pedagogía no lineal. De hecho, la información proporcionada por la técnica de coordenadas polares puede ayudar a comprender el impacto que ejercen las tareas propuestas, pudiendo complementar a la obtenida mediante el uso de otras herramientas, habitualmente empleadas en el control de la carga de entrenamiento. Asimismo, al posibilitar el análisis de los comportamientos realizados en el contexto real de juego, podría ayudar a solventar los problemas de validez ecológica que presentan algunos de los test empleados para valorar la eficacia de las propuestas metodológicas empleadas (Camacho, 2008; Roberts, et al., 2019).

\section{Conclusiones}

En relación al objetivo principal del estudio y teniendo en cuenta los resultados de ambos grupos de manera conjunta, las conclusiones más importantes que se pueden extraer son las siguientes:

La utilización de la defensa zonal de una línea fomentó la realización de los siguientes comportamientos en ataque: fijación impar, bloqueo, lanzamiento tras penetración y lanzamiento a distancia.

La utilización de la defensa zonal de dos líneas con defensor avanzado dificultó la realización de habilidades colectivas en ataque y activó las pérdidas de balón. Asimismo, activó la realización de desmarques del pivote.

La utilización de la defensa zonal de una línea fomentó la realización de las siguientes habilidades defensivas: cambio de oponente, contrabloqueo, doblaje y unos contra uno.

La utilización de la defensa zonal de dos líneas con defensor avanzado fomentó la realización de estas habilidades defensivas: deslizamiento, interceptación y marcaje individual al pivote.

Estos comportamientos no se expresaron de la misma forma en ambos equipos. La influencia de los constreñimientos es específica para cada equipo y jugador, en función de sus constreñimientos individuales (capacidad para lanzar a distancia, capacidad para desmarcarse, capacidad para jugar con el pivote, etc.). Esto supone un desafío para los entrenadores, pues, por un lado deben conocer la influencia general ejercida por los constreñimientos empleados, a la vez que deben identificar las tendencias específicas que presenta un determinado jugador o un grupo de jugadores. Este hecho cuestiona la validez de recetas ideales para todos.

Finalmente, una de las limitaciones del presente estudio es estudiar el papel de los constreñimientos individuales. Por este motivo, sería interesante investigar la influencia ejercida por los constreñimientos estudiados en jugadores de diferente edad o diferente nivel de habilidad. Por otro lado, aunque los juegos reducidos son representativos del juego real y permite la transferencia de los aprendizajes adquiridos presentan algunos inconvenientes. La tarea empleada en esta investigación, debido a sus dimensiones y a la forma del área, no ha permitido estudiar comportamientos que impliquen a los extremos. Consideramos que en futuros estudios sería interesante investigar la influencia de los constreñimientos estudiados en partidos desarrollados en espacios más o menos amplios. Asimismo, debido a la carencia de estudios que aborden la implantación de la pedagogía no lineal en la enseñanza del balonmano, sería recomendable la realización de más investigaciones sobre la influencia que ejercen otros constreñimientos en los comportamientos que se dan en las distintas fases del juego.

\section{Referencias}

Anguera, M. T. (2003). La observación. En C. Moreno Rosset (Ed.), Evaluación psicológica. Concepto, proceso y aplicación en las áreas del desarrollo y de la inteligencia (pp. 271-308). Madrid: Sanz y Torres.

Anguera, M. T., Blanco-Villaseñor, A., Hernández-Mendo, A., \& Losada, J. L. (2011). Diseños observacionales: Ajuste y aplicación en psicología del deporte. Cuadernos de Psicología del deporte, 11(2), 63-76.

Anguera, M. T., \& Hernández-Mendo, A. (2013). La metodología observacional en el ámbito del deporte. E-Balonmano.com: Revista de Ciencias del Deporte, 9(3), 135-160.

Anguera, M.T., Magnusson, M.S., \& Jonsson, G.K. (2007). Instrumentos no estándar. Avances en Medición, 5(1), 63-82.

Antón, J.L. (2000). Balonmano: Perfeccionamiento e investigación. Barcelona: Inde.

Ávila, F.M. (2015). Defender al pivote atacante: Trabajo global del equipo. E- balonmano.com: Revista de Ciencias del Deporte 11(2),143-166.

Ávila-Moreno, F. M., Chirosa-Rios, L.J., Ureña-Espá, A., Lozano-Jarque, D. \& Ulloa-Díaz, D. (2018). Evaluation of tactical performance in invasion team sports: a systematic review. International Journal of Performance Analysis in Spor 18(2), 
195-216. https://doi.org/10.1080/24748668.2018.1460054

Bakeman, R. \& Quera, V. (2011). Sequential analysis and observational methods for the behavioral sciences. Cambridge: Cambridge University Press. https://doi.org/10.1017/ CBO9781139017343

Balagué, N., Pol, R., Torrents, C., Ric, A., \& Hristovski, R. (2019). On the Relatedness and Nestedness of Constraints. Sports Medicine, 5(6), 1-10. https://doi.org/https://doi.org/10.1186/ s40798-019-0178-z

Balagué, N., Torrents, C., Pol, R., \& Seirul·lo, F. (2014). Entrenamiento integrado. Principios dinámicos y aplicaciones. Apunts Educación Física y Deportes, 116(2), 60-68. https://doi.org/ DOI: http://dx.doi.org/10.5672/apunts.2014-0983.es.(2014/ 2). 116.06

Cardinet, J., Johnson, S., \& Pini, G. (2010). Applying Generalizability Theory using EduG. New York: Routledge

Castañer, M., Barreira, D., Camerino, O., Anguera, M. T., Canton, A., \& Hileno, R. (2016). Goal scoring in soccer: A polar coordinate analysis of motor skills used by Lionel Messi. Frontiers in Psychology, 7(MAY), 1-10. https://doi.org/ 10.3389/fpsyg.2016.00806

Castañer, M., Barreira, D., Camerino, O., Anguera, M. T., Fernandes, T., \& Hileno, R. (2017). Mastery in goal scoring, T-pattern detection, and polar coordinate analysis of motor skills used by Lionel Messi and Cristiano Ronaldo. Frontiers in Psychology, 8(MAY). https://doi.org/10.3389/ fpsyg.2017.00741

Camacho, P. (2008). Influencia de una estrategia de enseñanza incidental sobre variables psicológicas, fisiológicas y motoras en jugadores de baloncesto de diferentes edades y niveles de peripecia (Tesis Doctoral). Universidad de Huelva, Huelva.

Cantos, J., \& Moreno, F. J. (2019). Pedagogía no lineal como método de enseñanza de los comportamientos tácticos en los deportes de equipo, aplicación al rugby. Retos, 35(1), 402-406

Chow, J. Y., Davids, K., Button, C., \& Renshaw, I. (2015). Nonlinear Pedagogy in Skill Acquisition: An Introduction. Abingdon: Routledge.

Chow, J. Y., Davids, K., Button, C., Shuttleworth, R., Renshaw, I., \& Araújo, D. (2007). The Role of Nonlinear Pedagogy in Physical Education. Review of Educational Research, 77(3), 251-278. https://doi.org/10.3102/003465430305615

Correia, V., Carvalho, J., Araújo, D., Pereira, E., \& Davids, K. (2018). Principles of nonlinear pedagogy in sport practice. Physical Education and Sport Pedagogy, 24(2), 117-132. https:/ /doi.org/10.1080/17408989.2018.1552673

Cronbach, L. J., Gleser, G. C., Nanda, H., y Rajaratnam, N. (1972). The dependability of behavioral measurements: Theory of generalizability for scores and profiles. New York: John Wiley and Sons.

Flores Rodríguez, J., \& Anguera, M. T. (2018). Patrón de juego en balonmano según el jugador que ocupa la posición de central. Apunts Educación Física y Deportes, 134(4), 110-123. https:/ /doi.org/DOI: http://dx.doi.org/10.5672/apunts.20140983.es.(2018/4).134.08

Anguera, M. T., \& Hernández-Mendo A. (2013). La metodología observacional en el ámbito del deporte. E-balonmano. com: Revista de Ciencias del Deporte, 9(3), 135-160.

Hernández-Mendo, A., López, J. A., Castellano, J., Morales, V., \& Pastrana, J. L. (2012). HOISAN 1.2: Programa informático para uso en Metodología Observacional. Cuadernos de Psicología del Deporte, 12(1), 55-78. https://doi.org/10.4321/S157884232012000100006

Jiménez-Salas, J., Morillo-Baro, J. P., Reigal, R. E., MoralesSánchez, V., \& Hernández-Mendo, A. (2020). Análisis de coordenadas polares para el estudio de los sistemas defensivos en balonmano. Cuadernos de Psicologia del Deporte, 20(1), 103117. https://doi.org/10.6018/cpd.396431

Landis, J. R., \& Koch, G. G. (1977). The Measurement of Observer Agreement for Categorical Data. Biometrics, 33(1), 159-174.

Lozano, D. (2014). Análisis del comportamiento táctico ofensivo en alto rendimiento en balonmano (Tesis doctoral). Universitat de Lleida. Lleida.

Lozano, D., Camerino, O., \& Hileno, R. (2016). Interacción dinámica ofensiva en balonmano de alto rendimiento. Apunts. Educación Física y Deportes, 125(3), 90-110. https://doi.org/ 10.5672/apunts.2014-0983.es.(2016/3).125.08

Montoya, M. (2010). Análisis de las finalizaciones de los jugadores extremo en balonmano (Tesis doctoral).Universitat de Barcelona, Barcelona.

Paulo, A., Infante, J., \&Araújo, D. (2019). A influência da pedagogia não-linear e da abordagem baseada nos constrangimentos no treino do remate no voleibol. Retos, 36, 590-596.

Práxedes, A. (2018). El diseño de tareas para el aprendizaje de las habilidades en el fútbol de iniciación. una aplicación desde las perspectivas cognitiva y ecológica (Tesis doctoral). Universidad de Extremadura, Cáceres.

Prudente, J. N., Cardoso, A. R., Rodrigues, A. J., \& Sousa, D. F. (2019). Analysis of the Influence of the Numerical Relation in Handball During an Organized Attack, Specifically the Tactical Behavior of the Center Back. Frontiers in Psychology, 10:2451. https://doi.org/10.3389/fpsyg.2019.02451

Prudente, J., Garganta, J., \& Anguera, M. T. (2004). Desenho e vali- dação de um sistema de observação no Andebol. Revista Portuguesa de Ciências do Desporto, 4(3), 49-65

Renshaw, I., \& Chow, J. Y. (2018). A constraint-led approach to sport and physical education pedagogy. Physical Education and Sport Pedagogy, 24(2), 103-116. https://doi.org/10.1080/ 17408989.2018.1552676

Renshaw, I., Chow, J. Y., Davids, K., \& Hammond, J. (2010). A constraints-led perspective to understanding skill acquisition and game play: A basis for integration of motor learning theory and physical education praxis? Physical Education \& Sport Pedagogy, 15, 117-137. https://doi.org/10.1080/ 17408980902791586

Roberts, S. J., Rudd, J. R., \& Reeves, M. J. (2019). Efficacy of using non-linear pedagogy to support attacking players' individual learning objectives in elite-youth football: Arandomised cross-over trial. Journal of Sports Sciences, 38(11-12), 14541464. https://doi.org/10.1080/02640414.2019.1609894

Román, J.D. (2005). Conceptos de ataque frente a las variantes defensivas 6:0 y 5:1. E- balonmano.com: Revista de Ciencias del Deporte 1, 3-16.

Román, J.D. (2016). Evolucion Del Juego En Defensa En Balonmano: Hacia Las Defensas Alternativas Como Concepto. E- balonmano.com: Revista de Ciencias del Deporte 12(3), 151-164.

Sackett, G. P. (1980). Lag Sequential Analysis as a data reduction technique in social interaction research. En D. B. Sawin, R. C. Hawkins, L. O. Walker, \& J. H. Penticuff(Eds.), Exceptional infant. Psychosocial risks in infant- environment transactions (pp. 300-340). New York: Brunner/Mazel.

Serra-Olivares, J. \& García-Rubio, J. (2017). La problemática táctica, clave en el diseño representativo de tareas desde el enfoque de la pedagogía no lineal aplicada al deporte. Retos, 32, 278-280.

Sousa, D. J., Prudente, J. N., Sequeira, P., López-López, J. A., \& Hernández-Mendo, A. (2015). Análisis de las situaciones de juego 2 vs2 en el campeonato europeo masculino de balonmano 2012: Aplicación de la técnica de coordenadas polares. Cuadernos de Psicologia del Deporte, 15(1), 181-194. https:// doi.org/10.4321/s1578-84232015000100018 